\title{
A Medical Image Enhancement Algorithm Based on Fuzzy Set Theory and Its GUI Implementation
}

\author{
Wen $\mathrm{He}$ \\ Chengdu medical college, Chengdu, Sichuan, 610500, China \\ hewen41731@163.com
}

Keywords: Fuzzy Set; Medical Image; Enhancement; GUI

\begin{abstract}
In this paper, a medical image enhancement algorithm based on fuzzy set theory is proposed. The algorithm takes the Pal-King fuzzy enhancement algorithm as the basis, and using one kind of sinusoidal function as the improved membership function to solve the problem of low gray information loss. This membership function has the adjustable parameter $k$, which can change the shape of the function according to the processing needs. The algorithm has been simulated in the MATLAB environment, and the graphical user interface is designed for it. Experiments show that the algorithm can effectively enhance the brightness and contrast of the medical image, highlight the edge and details of the image, and has a certain application value and significance.
\end{abstract}

\section{Introduction}

In clinical diagnostics, medical image is an important tool to assist doctors to diagnose, such as X-ray, ultrasound, CT, MRI and so on, which are widely used in all aspects of medical diagnosis. But in the imaging process of the medical image, due to some external factors including the quality of imaging equipment, the operator's experience level, the pathological conditions of some patients and so on may result in poor image quality, such as the details are not clear enough, low contrast, blurry and so on, which affect the doctor's diagnosis and analysis. At this time, we can enhance these images by some image processing methods, in order to improve the quality and the visual effect of the image.

At present, there are many mature and effective image enhancement algorithm is applied in medical image processing, such as grayscale transformation, histogram equalization (HE) and contrast limited adaptive histogram equalization(CLAHE) in spatial domain; high-pass filtering, high frequency emphasis filtering and homomorphic filtering in frequency domain; wavelet enhancement and wavelet threshold denoising in wavelet domain, etc. These algorithms are able to effectively enhance the visual effect of medical images, but the emphasis of each algorithm is different, which has its own advantages and disadvantages and application scope. In the middle of 1980s, Pal and King proposed a fuzzy domain enhancement algorithm for image [1].Since then, the fuzzy theory has been widely applied to the field of image enhancement, and have achieved good results. However, the traditional Pal-king algorithm has the problem of losing partial details of the image [2]. This paper proposes an improved algorithm for this problem, and design and implements the GUI of the algorithm in MATLAB. Experiments show that the proposed algorithm can effectively enhance the medical images, from the GUI of the algorithm, we can easily adjust the parameters to achieve interactive image enhancement.

\section{Pal-King Fuzzy Enhancement Algorithm}

In Pal-King algorithm, an image of size $M \times N$ and $L$ gray levels is represented as a fuzzy matrix of size $M \times N \quad[3]$ : 


$$
X=\left[\begin{array}{cccc}
\mu_{11} / x_{11} & \mu_{12} / x_{12} & \cdots & \mu_{1 N} / x_{1 N} \\
\mu_{21} / x_{21} & \mu_{22} / x_{22} & \cdots & \mu_{2 N} / x_{2 N} \\
\cdots & \cdots & \cdots & \cdots \\
\mu_{M 1} / x_{M 1} & \mu_{M 2} / x_{M 2} & \cdots & \mu_{M N} / x_{M N}
\end{array}\right]
$$

In Eq. $1, \mu_{i j} / x_{i j}$ is the membership degree of the image pixel $(i, j)$ relative to a particular gray level. In Pal-King's algorithm, its membership function is used:

$$
\mu_{i j}=T\left(x_{i j}\right)=\left(1+\left(x_{\max }-x_{i j}\right) / F_{d}\right)^{-F_{e}} \text {. }
$$

In Eq. 2, $\mu_{i j}$ is the fuzzy characteristic value after the image transformation, $x_{\max }$ is the maximum value of the pixels in the image, $F_{e}$ is an exponential type fuzzy factor, its value is usually taken 2. $F_{d}$ is the reciprocal type fuzzy factor, and its value can be determined by $F_{e}$ and crossover point. When $X_{i j}=X_{c}$, if there is $T\left(X_{c}\right)=0.5$, then $X_{c}$ is called the crossover point.

After calculating the $\mu_{i j}$, and then to enhance the fuzzy characteristic value of the image, the membership function is defined as follows:

$$
I\left(\mu_{i j}\right)=\left\{\begin{array}{cl}
2 \mu_{i j}^{2} & 0<\mu_{i j}<0.5 \\
1-2\left(1-\mu_{i j}\right)^{2} & 0.5 \leq \mu_{i j} \leq 1
\end{array} .\right.
$$

Fuzzy characteristic value $\mu_{i j}$ perform $n$ times $I$ transformation,and the fuzzy set after transformation is obtained.

$$
\mu_{i j}^{\prime}=I_{n}\left(\mu_{i j}\right)=I\left(I_{n-1}\left(\mu_{i j}\right)\right) \text {. }
$$

Finally, the enhanced $\mu_{i j}^{\prime}$ to carry on the inverse transformation, and the fuzzy enhanced image $X^{\prime}$ is obtained, the gray value of its $\operatorname{pixel}(i, j)$ is:

$$
x_{i j}^{\prime}=T^{-1}\left(\mu_{i j}^{\prime}\right) \text {. }
$$

Wherein $T^{-1}(\cdot)$ is the inverse tranformation of Eq. 2. The new fuzzy feature plane can be generated by the Eq. 4, in a finite number of iterations, the image can be effectively enhanced. This is the classic Pal-King algorithm.

However, in the classical Pal-King algorithm, there are some shortcomings. As shown in Fig. 1, in a given $X_{c}$ and $F_{e}$, we can draw the mapping curve of the $\mu_{i j}$ and $x_{i j}$. It can be seen that when $x_{i j}=0, \mu_{i j}=\left(1+x_{\max } / F_{d}\right)^{-F_{e}}$, its value is not 0 , that is $\mu_{\text {Min }}$ is not zero. Then after the membership values of the image perform $I_{n}$ transformation, there will be a part of the $\mu_{i j}$ transformed to a region less than $\mu_{\text {Min }}$, which makes the inverse transformation of Eq. 2 will occur no solution phenomenon. In order to ensure $T^{-1}(\cdot)$ has a solution, when $\mu_{i j}^{\prime}<\mu_{\text {Min }}$, the algorithm mandatory $\mu_{i j}^{\prime}=\mu_{M i n}$, this will result in the original image, most low grayscale is mandatory to zero in the enhanced image, resulting in the loss of part of the gray information, affecting the enhanced image detail and edge detection [4].

In addition, the crossover point is selected by experience or multiple attempts to obtain, which is random, and the selection of different thresholds has a great influence on the enhancement effect of the image. In the algorithm of Pal-King, there is not a set of mature and feasible crossover point selection scheme [5]. 


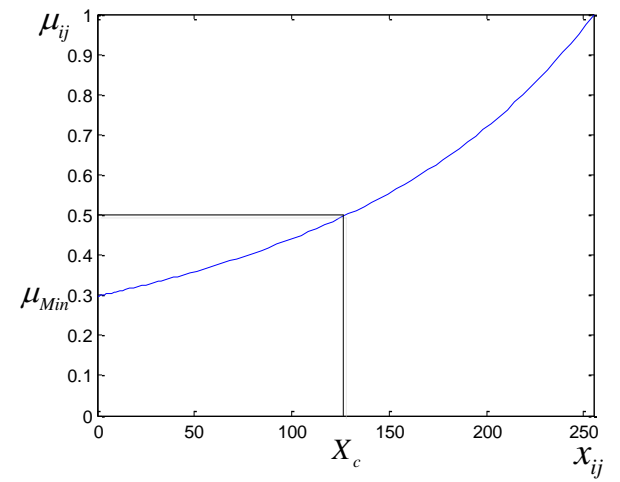

Figure 1. Mapping curve of $\mu$ and $x$ in P-K algorithm Figure 2. Mapping curve of $\mu$ and $x$ in proposed algorithm

\section{Improved Fuzzy Enhancement Algorithm}

In this paper, based on the Pal-King algorithm, we introduce a sinusoidal function as the membership function, and the function is defined as follows [6]:

$$
\mu_{i j}=\left[\sin \left(\frac{x_{i j}-x_{M i n}}{x_{M a x}-x_{M i n}} \times \pi / 2\right)\right]^{k} .
$$

Wherein, $x_{\text {Max }}$ is the maximum gray value of the image, $x_{\text {Min }}$ is the minimum gray value of the image, since the scope of the mapping function is $[0,1]$, so there won't appear the grayscale mandatory cut phenomenon. Meanwhile,the shape of function can be regulated by the adjusting parameter $k$,as shown in Fig. 2, when the value of $k$ in the range [0,1],the shape of the function is shown in the three blue curves in Fig. 2, at this time the brightness of the image can be improved greatly; when the $k$ value is larger than 1 and gradually increases, the shape of the function is shown in the four red curves in Fig. 2,gradually approaching the S-type, at this time it can reduce the membership of dark pixels and improve the membership of bright pixels, in order to enhance the contrast of the image in transformation.

After the image is mapped to the fuzzy domain through improved membership function, and then using the Eq.3 to perform fuzzy enhancement processing on the image. Finally the image is inverse transformed from fuzzy domain to the spatial domain so as to obtain the final enhanced image. The inverse transformation formula is :

$$
x_{i j}^{\prime}=x_{M \text { in }}+\left(x_{M \text { ax }}-x_{M \text { in }}\right) \cdot \arcsin \left(u_{i j}^{\prime k^{-1}}\right) \cdot(2 / \pi) .
$$

\section{Experimental Results and Analysis}

This paper selects a cardiac ultrasound image as the test object, as shown in Fig. 3, the size of $504 \times 367$, gray level is $0-255$, and the algorithm is simulated on MATLAB 7.9 platform. The iteration times of all algorithms are set to 1. Fig. 4 is the processing results of the classical Pal-King algorithm, its parameters are set to $F_{e}=1, F_{d}=128$, it can be seen that a part of gray information in image is lost,so it looks relatively dark. Fig. 5 is the result of the proposed algorithm when $\mathrm{k}=0.3$, it can be seen that the image brightness has been greatly improved, many details of the image is to show up. Fig. 6 is the result of the proposed algorithm when $\mathrm{k}=1.2$, it can be seen that the contrast of the image has been greatly improved,the brightness control is relatively ideal,and the left ventricle boundary of the cardiac ultrasound image is highlighted,this will greatly help for the left ventricle segmentation and measurement in future. 


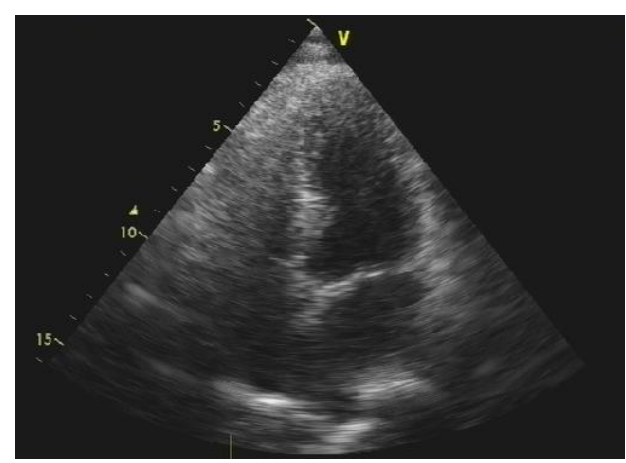

Figure 3. Original image

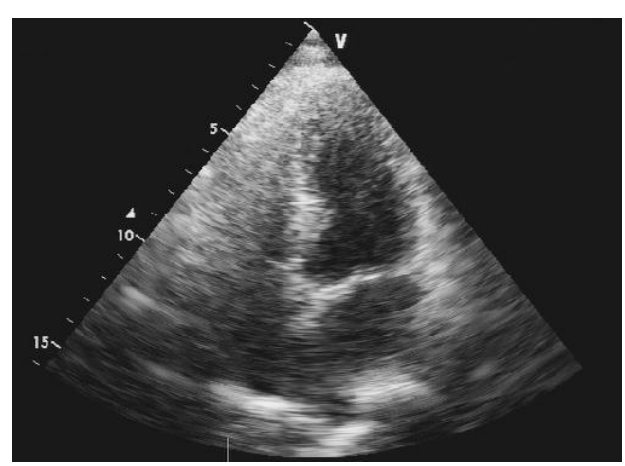

Figure 5. Proposed algorithm when $\mathrm{k}=0.3$

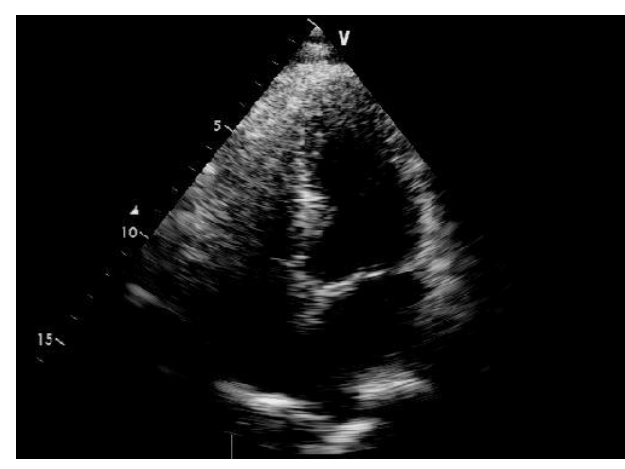

Figure 4. P-K algorithm

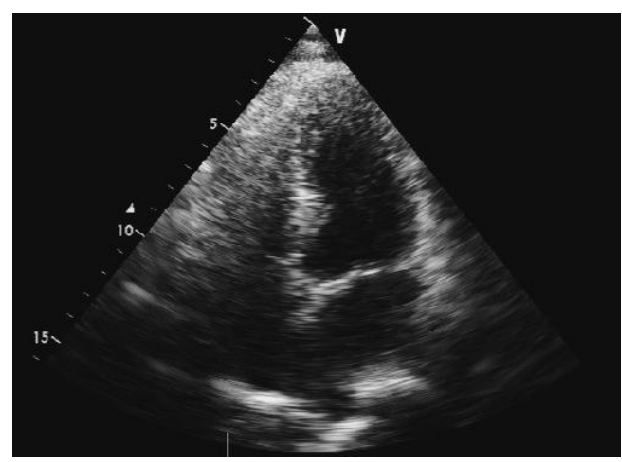

Figure 6. Proposed algorithm when $\mathrm{k}=1.2$

\section{The Implementation of Algorithm's GUI}

Since the selection of adjusting parameter $k$ value has a great influence on the image enhancement effect,so it is necessary to design an algorithm GUI to observe the image enhancement effect interactively. This paper designed the GUI of the algorithm in MATLAB environment, as shown in figure 7 :

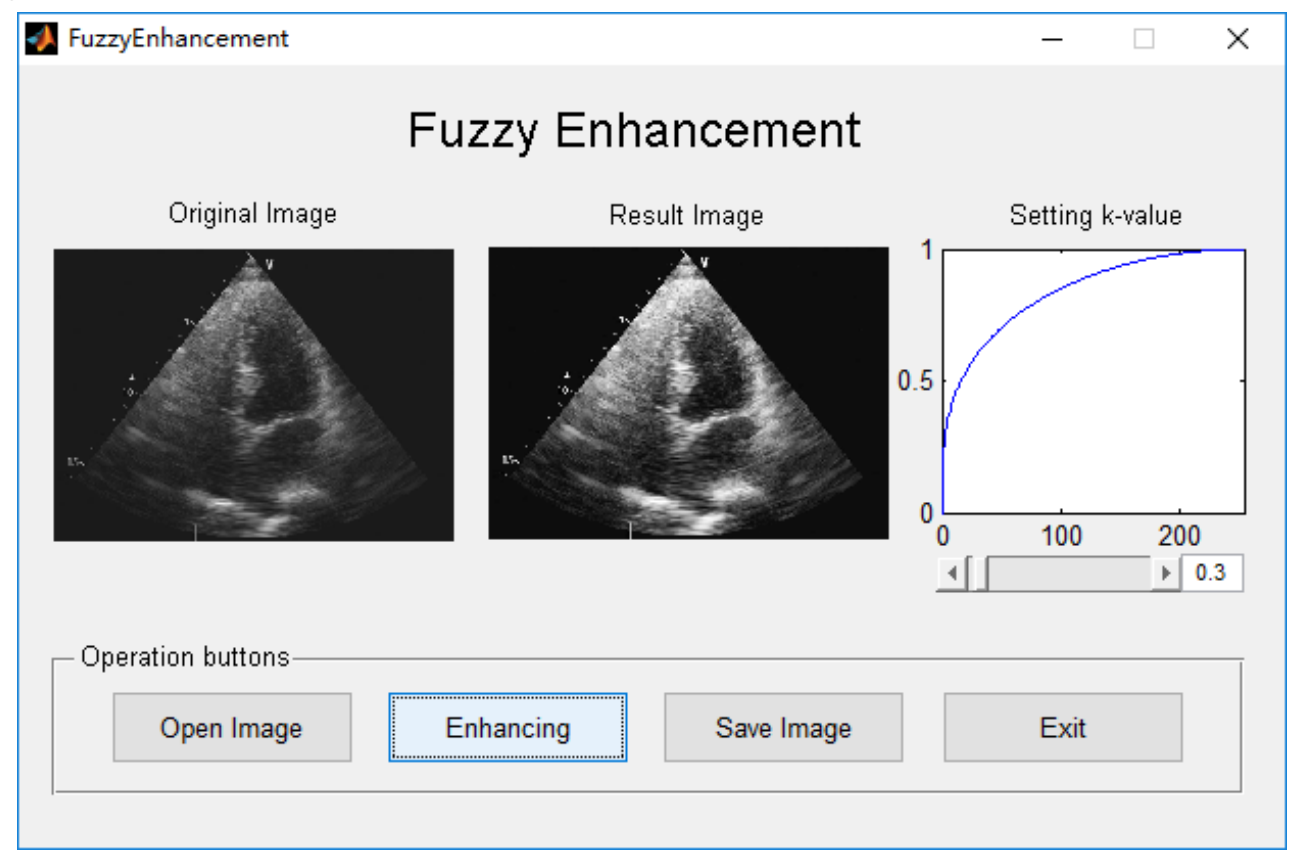

Figure 7. GUI design of the Algorithm

The design steps of the GUI of the algorithm are as follows:

1) Create the main interface of the program by MATLAB GUIDE,add the necessary controls on the main interface,such as the three Axes used to display the original image,enhanced image and membership function curve; a Slider and a Edit Text used to adjust the $k$ parameter settings; the four Push Button used to open the image, enhance the image, save the image and exit the program. 
2) Reasonable layout the controls,set the properties of each control, including the value of each control's Tag, String value, size, location and appearance properties.

3) Write the callback function of each control, The GUI is via the callback function to complete the event-driven, such as the click of a button,slide a scrollbar and so on. Wherein the callback function of the "Open Image" button is as follows:

[B,PathA]=uigetfile(\{'*.bmp';'*.jpg';'*.tif';'*.gif';'*.png'.... $\},$ 'open image');

$\mathrm{c}=[$ PathA,B];

axes(handles.axes1);

$\mathrm{F}=\mathrm{imread}(\mathrm{c})$;

imshow $(\mathrm{F})$;

4) Debug and run the program, generate .Fig and .m files.

5) Compile the .Fig and .M files generated by step 4 into an application that can be run independently, and then complete the design of GUI. Fig.7 shows the enhancement result of a cardiac ultrasound image when the adjusting parameter $k$ is set to 0.3 .

\section{Conclusions}

This paper proposed a medical image enhancement algorithm based on fuzzy set theory,the algorithm takes the Pal-King fuzzy enhancement algorithm as the basis, and using one kind of sinusoidal function as the improved membership function which can effectively avoid the problem of low grayscale being cut. At the same time,the different values of adjusting parameters can bring the different enhancement effect to image, when the value of $k$ is in the range[0,1], it can enhance the brightness of the image; when the value of $k$ is greater than 1,it can enhance the contrast of the image and highlight the image details. We designed the GUI of the algorithm in MALTAB,which can easily adjust the value of the parameter $k$ and observe the image enhancement effect. Experiments show that the algorithm can effectively enhance the medical image, and has a certain application value.

\section{Acknowledgement}

This work is supported by Sichuan Education Department Science Project (No. 16ZB0283) and Chengdu Medical College Science Project (No. CYZ11-018).

\section{References}

[1] S.K. Pal and R.A. King. Image Enhancement Using Smoothing with Fuzzy Sets. IEEE Transactions on Systems, Man and Cybernetics, 1981, 11(7):494-501.

[2] C.H. Zhao and Z.L. Liu. Image enhancement algorithm based on fuzzy set theory. Journal of Natural Science of Heilongjiang University, 2012, 29(3):401-406.

[3] Q.W. Jiang. Theory based on fuzzy image enhancement technology research and implementation (MS., East China Normal University, China 2009), p.26-32.

[4] H.T. Yu. Research on Image Enhancement Algorithms Based on Fuzzy Set Theory (MS., Xi`an University of Science and Technology, China 2005), p.11-12.

[5] Y. Yang and S.Y. Huang. Modified Pal and King algorithm for fuzzy edge detection. Chinese Journal of Scientific Instrument, 2008, 29(9):1918-1922.

[6] Y.Y. Cai, L.P. Xu, C.Y. Guo and Y.M. Wu. Application of Fuzzy Logic to the Enhancement of Pneumoconiosis X-ray Films. Microcomputer Information, 2008, 24(4-3):290-291. 